\title{
REVIEW
}

\section{Bacterial toxin-antitoxin systems targeting translation}

\author{
Ramón Díaz-Orejas, Elizabeth Diago-Navarro, Ana María Hernández Arriaga, Juan López-Villarejo, \\ Marc Lemonnier, Inma Moreno-Córdoba, Concha Nieto, Manuel Espinosa
}

Departamento de Microbiología Molecular y Biología de las Infecciones, Centro de Investigaciones Biológicas CSIC, Madrid, Spain

Received $5^{\text {th }}$ March 2010.

Revised 30 ${ }^{\text {th }}$ April 2010.

Published online $17^{\text {th }}$ May 2010.

\begin{abstract}
Summary
Toxin-antitoxin systems (TAS) emerged more than 25 years ago and have since developed as an important field in molecular microbiology. TAS are autoregulated operons coding a stable toxin and an unstable antitoxin found in the plasmids and chromosomes of Bacteria and Archaea. The conditional activation of their toxins interferes with cell growth/viability and, depending on the context, can influence plasmid maintenance, stress management, bacterial persistence, cell differentiation and, it is likely also bacterial virulence. This review summarizes recent results on the parD system of plasmid R1 and on the chromosomal relBE systems found in Escherichia coli and in Streptococcus pneumoniae with a focus on the RNase activity of their toxins, their regulation and their biomedical applications and implications.
\end{abstract}

Key words: toxin-antitoxin systems; plasmid maintenance; cell growth regulation; toxin RNases; protein synthesis inhibition

\section{INTRODUCTION}

Toxin-antitoxin systems (TAS) were reported more than 25 years ago as plasmid auxiliary maintenance cassettes. Three plasmid-borne TAS contributed initially to establishing this field in microbiology: the parB (hok, sok) and parD (kis, kid) systems of plasmid R1 and the $c c d$ ( $c c d A, c c d B$ ) system of plasmid F (Gerdes et al. 1986, Hiraga et al. 1986, Bravo et al. 1987). TAS are based on the activity of two intracellular components, a stable toxin and a

\begin{tabular}{|c|c|}
\hline$\triangle$ & Ramón Díaz-Orejas, Centro de \\
\hline & Investigaciones Biológicas (CSIC), Ramiro \\
\hline & $\begin{array}{l}\text { de Maeztu 9, E-28040 Madrid, Spain } \\
\text { ramondiaz@cib.csic.es }\end{array}$ \\
\hline & $+34-91-8373112$ \\
\hline & $+34-91-5360432$ \\
\hline
\end{tabular}

constitutively unstable antitoxin; the differential decay of the antitoxin, occurring also in cells losing the plasmid at cell division, allows activation of the toxin in these cells and its subsequent interference with cell growth/viability. This favours the preferential propagation of plasmid containing TAS and contributes to their maintenance in bacterial populations. The three systems described, initially define two main TAS types: hok/sok is the prototype of Type I TAS in which the toxin is a protein and the antitoxin is an RNA, while $c c d$ and parD define type II TAS in which both the antitoxin and the toxin are proteins. Most of the focus has been so far concentrated on type II systems (Hayes 2003, Buts et al. 2005, Gerdes et al. 2005, Condon 2006, Kamphuis et al. 2007a, Van Melderen and Saavedra De Bast 2009).

Following the initial reports, many type II systems were found in plasmids and also in the chromosomes of many Eubacteria and Archaea (Pandey and Gerdes 
2005). Up to 9 different type II TA families have been defined. TA systems can contribute to stress management in bacterial populations, to genome stability or to complex phenotypes such as bacterial persistence and cell differentiation and eventually as anti-addiction modules (Van Melderen and Saavedra De Bast 2009).

This review summarizes recent contributions made in our two groups (RDO and MEP) on the TAS systems parD (kis, kid) of plasmid R1 and relBE of Streptococcus pneumoniae, whose toxins cleave mRNAs. We focus on the mechanisms of action of the toxins of these TAS which inhibit translation, and on the way they are regulated. The biomedical implications/applications of these studies is briefly addressed.

\section{THE parD SYSTEM OF PLASMID R1: INHIBITION OF PROTEIN SYNTHESIS BY mRNA CLEAVAGE IN THE ABSENCE OF RIBOSOMES}

Identification of the parD system and its specific role in the maintenance of plasmid $\mathrm{R} 1$

parD (kis, kid) system was reported by our laboratory in 1987 (Bravo et al. 1987). This TAS is a bicistronic operon of plasmid R1 which is close to the basic replicon of the plasmid. It codes two short proteins, an antitoxin, Kis (killer suppressor), and a toxin, Kid (killing determinant). An identical system to parD, pem, was also found in plasmid R100 (Tsuchimoto et al. 1988) and two chromosomal TA systems homologous to pem (chpA/mazEF and $c h p B$ ) were later identified by the same authors (Masuda et al. 1993). A marginal cross-talk between these systems and parD that could be enhanced by mutations affecting the chpAI/mazE and chpBI antitoxins could be detected (Santos-Sierra et al. 1997, 1998). Under standard growth conditions parD is inefficient as a plasmid maintenance system and for this reason it was initially undetected. It was identified by an unexpected mutation in the kis antitoxin gene that greatly increased the stability of the plasmid and in combination with other mutations, revealed the toxin and antitoxin activities of Kis and Kid (Bravo et al. 1987, 1988). The first kis mutant also indicated a key role for the antitoxin in the transcriptional regulation of the parD operon. Unexpectedly, the parD system was activated when replication of a low copy mini-R1 plasmid was compromised and, surprisingly, this activation contributed to recover the plasmid replication (Ruiz-Echevarría et al. 1995b). This result suggested the specialized role of the system in recovering inefficient plasmid replication, and this recovery was clarified following the identification of the RNase activity of Kid (see below): it seems that the activated Kid toxin modulates the efficiency of replication by down-regulating the mRNA levels of CopB, the auxiliary R1 copy number controller, via specific RNA cleavage within the polycistronic copB-repA mRNA (Pimentel et al. 2005). These results suggested a functional coupling between the replication and parD modules in plasmid R1.

\section{The RNase activity of Kid toxin}

The discovery that RelE toxin belonging to the relBE system inhibits translation by cleaving the mRNA on the ribosome (Pedersen et al. 2003), triggered a search for similar activities in the toxins of other TAS. It was found that Kid/PemK, the identical toxin of the parD/pem systems of plasmid R1 and R100 (Tsuchimoto et al. 1988), are specific endoribonucleases that, in contrast to RelE, can cleave RNA in the absence of ribosomes (Zhang et al. 2003, Zhang et al. 2004, Muñoz-Gómez et al. 2004, Muñoz-Gómez et al. 2005). This cleavage inhibits the potential of the cells to synthesize proteins. Kid toxin cleaves RNA as RNase A or RNase T1 do (Kamphuis et al. 2006). This cleavage is initiated by a nucleophilic attack of the scissile phosphate by the oxygen of an adjacent 2'-OH residue and requires a catalytic acid, a catalytic base and additional stabilizing interactions. The structure of the functional dimeric Kid toxin is available (Hargreaves et al. 2002) and the model of a structure of the complex of Kid with a mimetic RNA substrate based in NMR data has been proposed (Kamphuis et al. 2006) (see Fig. 1). Residues proposed by this model to play key roles in RNA cleavage and in specific interactions with the substrate have been evaluated using a collection of specific mutants in the toxin (Diago-Navarro et al. 2009a). The results of these studies have supported the predictions of the model. Kis/PemI and MazE antitoxins are able to neutralize the RNase activity of their respective toxins in addition to their potential in inhibiting protein synthesis (Muñoz-Gómez et al. 2004, 2005, Zhang et al. 2003, 2004). The available structures of the $\mathrm{MazF}_{2}-\mathrm{MazE}_{2}-\mathrm{MazF}_{2}$ heterohexamer (Kamada et al. 2003) and of the $\mathrm{Kid}_{2}-\mathrm{Kis}_{2}-\mathrm{Kid}_{2}$ heterohexamer, modelled on the MazE-MazF heterohexamer (Kamphuis et al. 2007b), explain this neutralization as the result of an interference of the C-terminal region of the antitoxin with toxin residues involved in RNA binding or cleavage. Neutralization of Kid RNase activity by the Kis antitoxin occurs either in prokaryotic or eukaryotic cells (de la Cueva-Méndez et al. 2003, Muñoz-Gómez et al. 2005). 
A

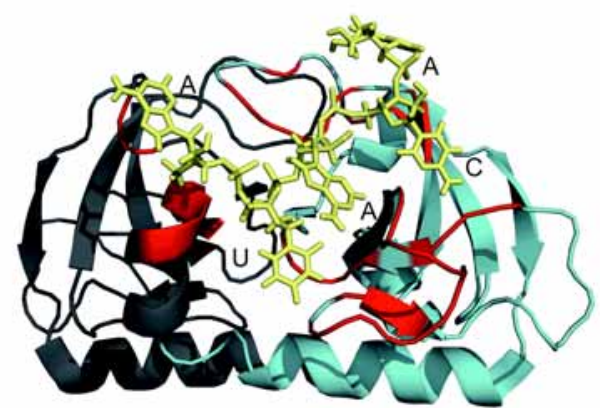

B

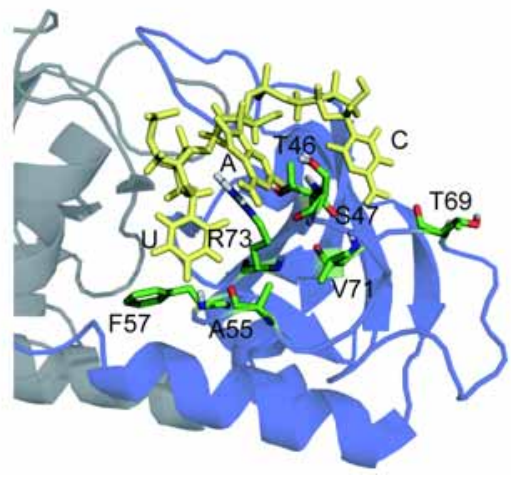

C

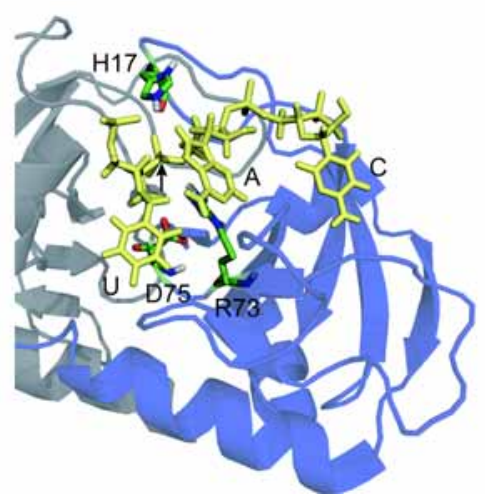

Fig. 1. Modelled mechanism of binding and cleavage of RNA by Kid toxin. A) RNA binding pocket of Kid. Kid monomers are coloured in grey and cyan. Residues involved in one of the symmetric RNA binding surfaces are shown in red. B) and C) show respectively Kid residues (sticks) involved in specific interactions with the RNA core sequence and these forming the active site of the protein. The arrow points to the scissile phosphate. RNA bases (AUACA) are coloured in pale yellow.

The RNase activity of Kid toxin explains several other results obtained before this primary activity was discovered: the recovery of compromised plasmid R1 replication was due to the RNase activity of the Kid toxin (Pimentel et al. 2005), and the inhibition of ColE1 replication by the Kid toxin observed in vitro and in vivo (Potrykus et al. 2002, Muñoz-Gómez et al. 2005) could be explained by the cleavage of the RNA transcript required to make the RNA that primes replication of this plasmid. This cleavage was shown using purified RNA ColE1 pre-primer as a substrate of Kid; however a detailed analysis of this cleavage occurring during transcription remains to be carried out. The ability of Kid to inhibit cell growth/viability both in prokaryotes and eukaryotes is related to the inhibition of protein synthesis due to its RNase activity (Muñoz-Gómez et al. 2005).

Interestingly the Kid toxin shows structural homology to the CcdB toxin (Hargreaves et al. 2002) even if CcdB rather than cleaving RNA targets DNA gyrase (Bernard and Couturier 1992).
Regulation of the parD system

TAS are subjected to strict control to prevent the deleterious effects of their toxins. The activity of the toxins is neutralized by direct interactions with their antitoxins (see above). Interestingly these interactions lead to complexes that regulate the operon and maintain, the toxin-antitoxin transcripts at low levels. Recent studies of the transcriptional regulation of parD showed that dimers of the antitoxin pilot the interactions of the repressor complex with specific sequences of the promoter-operator region and that the relative levels of Kis and Kid proteins influence this regulation: a hetero-octameric complex formed in excess of the antitoxin binds in the promoter-operator region efficiently, suggesting that it is involved in repression, and a heterohexameric Kis-Kid complex formed in excess of the toxin is the main species involved in toxin neutralization (Monti et al. 2007, Kamphuis et al. 2007b). This complex binds with low affinity to the promoter-operator region thus favouring deregulation of the operon and reposition of the antitoxin levels. The interplay of the two proteins 
could restore the regulated situation when the antitoxin decays. This hypothesis explains the requirement of two different proteins to regulate the system.

Post-transcriptional regulatory mechanisms (Ruiz-Echevarría et al. 1995a) contribute to maintain, under standard growth conditions, an $\mathrm{A} / \mathrm{T}$ ratio close to 2 that results in a repressed situation. The selective activity of the Lon protease on the Kis antitoxin (Tsuchimoto et al. 1992) can unbalance this situation.

\section{RelBE TYPE SYSTEMS: PROTEIN INHIBITION BY CLEAVAGE OF mRNA ON THE RIBOSOMES}

One of the most studied TAS members is the relBE gene, present in the chromosome of many eu- and archae- bacteria and firstly reported in E. coli (Christensen et al. 2001). Homologues were found in Bacteria, Archaea (Gerdes et al. 2005, Pandey and Gerdes 2005), and in plasmids (Gronlund and Gerdes, 1999, Hayes, 2003). In addition to the E. coli operon (Gotfredsen and Gerdes 1998), the nature as bona fide TAS relBE loci has been confirmed for S. pneumoniae (Nieto et al. 2006), Mycobacterium tuberculosis (Korch et al. 2009), Pyrococcus horikoshii (Takagi et al. 2005), and Methanococcus jannaschii (Francuski and Saenger 2009). In E. coli cultures subjected to nutritional stress, especially in conditions that impair protein synthesis, transcription of relBE increased and toxin RelE was activated due to degradation of its cognate RelB antitoxin by the Lon protease. This resulted in cell growth arrest, concomitantly with inhibition of translation (Christensen et al. 2001, Gotfredsen and Gerdes, 2002). Over-expression of EcRelE induced stasis from which cells can recover by antitoxin production (Pedersen et al. 2002). A similar behaviour was found in $E$. coli by activation of the pneumococcal SpRelE toxin but, in this case, prolonged exposure of the cells to the toxin led to cell death rather than to cell stasis (Nieto et al. 2006). Therefore, chromosomal relBE could act as a stress response locus more than a cell killing system, adjusting the rates of protein synthesis under unfavourable growth conditions.

\section{RelE mRNA cleavage mechanism}

RelE was shown to inhibit translation by cleaving mRNAs, both in vivo and in vitro by RelE positioning at the ribosomal A site (Christensen and Gerdes, 2003, Pedersen et al. 2003). Determination of the RelE structure showed that the protein has a shape similar to the C-terminal region of the translation elongation factor EF-G (Takagi et al. 2005), thus allowing its access into the A site of the ribosome. RelE-mediated cleavage occurred preferentially between the second and the third bases of stop and sense codons with a $G$ at third position (Pedersen et al. 2003). In agreement with the entrance of RelE in the ribosomal A-site was the finding that the release factor I (RFI), which binds to the ribosomal A site in the translation termination stage prevented in vitro cleavage of mRNA mediated by RelE (Pedersen et al. 2003). RFI-mutants showed increased sensitivity to the RelE and Kid toxins (Diago-Navarro et al. 2009b). In the case of RelE, overproduction of tmRNA was able to rescue stalled ribosomes, thus counteracting its toxic effect (Christensen and Gerdes 2003). EcRelE only cleaved ribosome-bound mRNAs (Christensen and Gerdes, 2003, Pedersen et al. 2003). However, E. coli strains with deletions in genes encoding toxins (RelE, MazF, ChpBK, YoeB, YafQ and $\mathrm{YhaV}$ ) were shown not to be involved in cleavage of a particular mRNA composed of rare Arg codons that caused ribosome pausing (Garza-Sánchez et al. 2008); in addition, arginine starvation was shown to induce mRNA cleavage at specific codons. However, cleavage occurred with the same specificity in the strain lacking the toxins, indicating that mRNA cleavage occurring during arginine starvation was independent of these known TA systems. Similar results were found by Aiba's group (Li et al. 2008b). Interestingly, comparison of the fitness of two isogenic E. coli strains, one wild type (wt) and the other having deletions in five TAS (mazEF, relBE, chpBK, yefM-YoeB, dinJ-yafQ) subjected to short-term stress conditions (amino acid starvation, acidic stress, antibiotic treatment, and long term stationary phase) showed no significant differences among them (Tsilibaris et al. 2007), suggesting that TAS could be involved only in long-term evolution (Van Melderen and Saavedra De Bast 2009).

Even though RelE has a microbial RNase fold it lacked both basic and acidic catalytic residues suggesting that they should be provided by the ribosome (Buts et al. 2005, Condon 2006). The solution of the three-dimensional structures of EcRelE, alone and bound to programmed Thermus thermophilus $70 \mathrm{~S}$ ribosomes in both pre- and post-cleavage states solved this puzzle (Neubauer, 2009). In the crystal, RelE occupies the A site, establishing direct contacts with the 16S rRNA, and thus preventing access of translation factors and tRNA to the ribosome. The overall structure of RelE hardly changed upon binding to the ribosome, but its interaction with the A site on the $30 \mathrm{~S}$ subunit seemed to reorganize the mRNA, promoting a 2'-OH hydrolysis between codon position two and three 


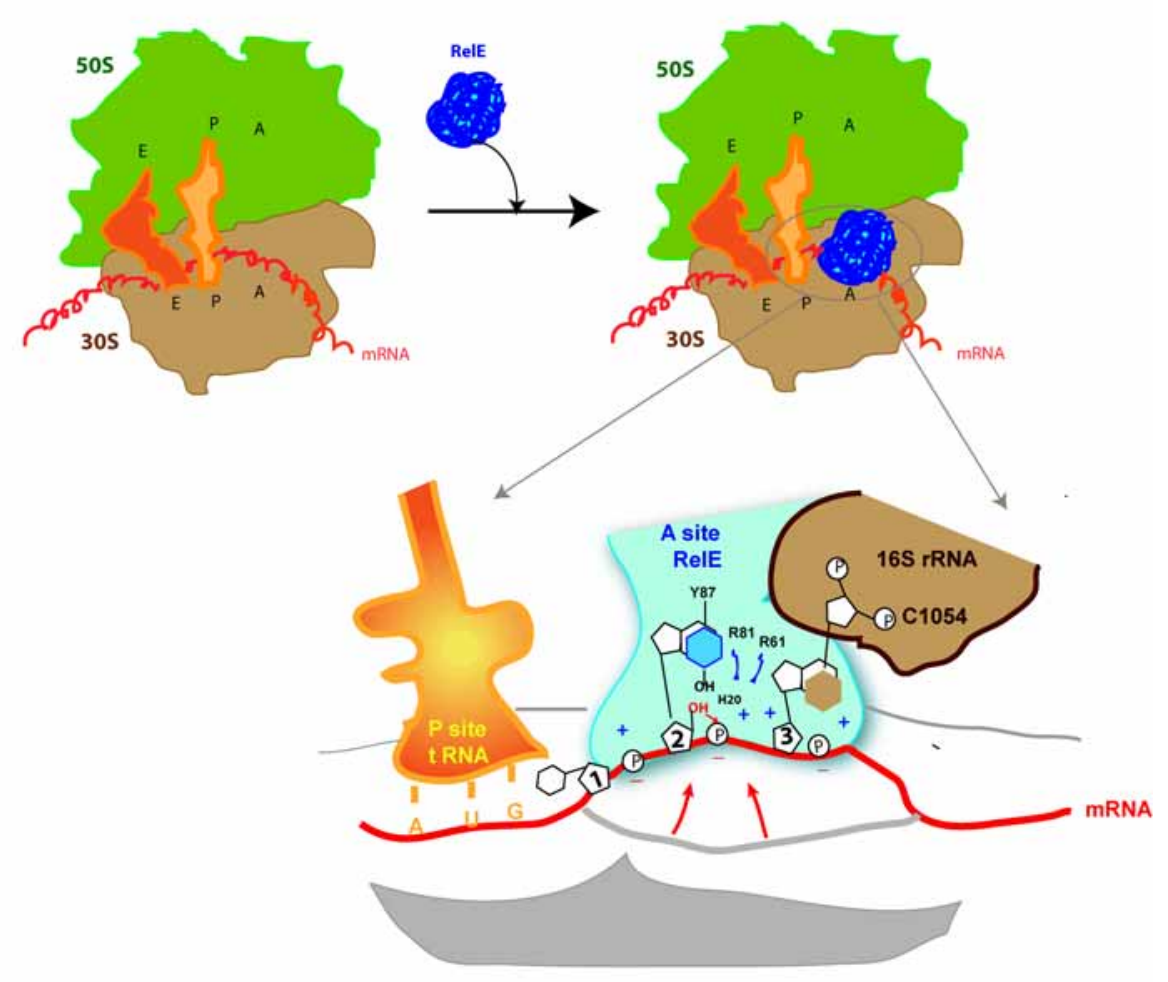

Fig. 2. Mechanism of cleavage of RNA on the ribosome. A) Schematic representation of RelE binding on the ribosome. E, P, and A denotes specific ribosomal sites involved in translation. B) Model of endonucleolytic reaction of RelE on translated mRNA. RelE approaches the mRNA to its active site through interactions between RelE basic side chains (+) (R45, R81, R61, K52, K54, and R56) and the phosphate group in the mRNA (-). The stacking of the second nucleotide with tyrosine 87(Y87) and of the third nucleotide with the residue C1054 of 16S rRNA are indicated. The red arrow indicates the nucleophilic attack of 2'O' on the phosphate group. The water molecule and residues R61 and R81 present in the active sites of RelE are also shown.

(Fig. 2). Considering the structural and functional data, a model for RelE-mediated cleavage of mRNA was proposed (Neubauer et al. 2009). Even though cleavage of the mRNA on the ribosome is carried out by RelE and not by the ribosome itself, this cell particle is essential for the RelE catalysis.

In addition to the $E$. coli RelE, the structure of the complex RelE-RelB, has been determined for P. horikoshii (PhRelBE) (Takagi et al. 2005) and M. jannaschii (MjRelBE) (Francuski and Saenger 2009). PhRelBE forms a tetrameric complex, in which a molecule of RelB wraps around a compact RelE dimer generating a heterodimer. Two heterodimers generate the heterotetramer through interactions between $P h$ RelB from one heterodimer and $P h$ RelE from another. The extensive wrapping of RelB around RelE makes the dimer bigger, thus hampering the entrance of RelE into the ribosome A-site. MjRelBE forms also a heterotetrameric complex with a different pattern. Data on the RelBE complex from E. coli indicate that the C-terminal region of RelB could be responsible for RelB-RelE interaction. This region showed high sensitivity to proteases, so that its interaction with RelE could confer resistance to degradation; thus the RelB C-terminal region could be involved in both self-association and RelE binding (Cherny et al. 2007). NMR studies showed that RelB-RelE complex provokes a helix displacement near the RelE mRNA interferase active site resulting in the neutralization of the positively charge catalytic sites by acidic residues from RelB antitoxin (Li et al. 2009). The E. coli RelB $\mathrm{N}$-terminal domain may form a dimeric ribbon-helix-helix structure (Cherny et al. 2007, Li et al. 2008a), making it likely that the antitoxin would use this motif for DNA binding ( $\mathrm{Li}$ et al. 2008a, Overgaard et al. 2008). However, the threedimensional structure of $P h$ RelB showed the antitoxin would recognize its DNA target as a dimer via a leucine zipper motif (Takagi et al. 2005). In conclusion, the structural data collected regarding RelB indicate that despite the sequence and structure 
homology between the toxins from E. coli, $M$. jannaschii and $P$. horikoshii, the antitoxins may differ in the mechanism they use to optimize their DNA binding and their stability properties, adapting them to their own host.

\section{Auto-regulation}

Two models have been proposed to explain the mechanism of autorepression of the E. coli relBE operon. The first considers the possibility that RelBE complex could bind as a tetramer to the operator sequence and that RelB has two levels of regulation (Li et al. 2008a). The second model (Overgaard et al. 2008) proposes that RelBE complex could control relBE transcription by binding to the DNA operator as a trimer, RelB $_{2}$-RelE. Thus, RelE would have two RelB binding sites, one of low and another of high affinity each playing different roles depending on the toxin:antitoxin ratios (Overgaard et al. 2008).

\section{YoeB mRNA interferase}

Another mRNA interferase homologous to RelE, YoeB, has been studied, albeit in less detail than RelE. It has been grouped as belonging to the RelE superfamily (Pandey and Gerdes 2005, Makarova et al. 2009), and studied in E. coli and in other microorganisms. Gene yoeB encodes toxin YoeB belonging to the TA pair YefMYoeB, which was identified as a homolog of the Txe toxin (Grady and Hayes 2003) in E. coli. The Axe-Txe TA pair was identified in a plasmid from Enterococcus faecium (Grady and Hayes 2003), and homologs have been studied in S. pneumoniae (Nieto et al. 2007), Staphylococcus aureus (Yoshizumi et al. 2009), and M. tuberculosis (Kumar et al. 2008). The structure of the $E$. coli YoeB and the complex with its cognate antitoxin, YefM, has been elucidated and the residues involved in YoeB catalysis have been identified. YoeB showed a structure similar to RNase Sa and Barnase, two proteins with a characteristic microbial RNase fold (Kamada and Hanaoka 2005); it also exhibited similarities with RelE from $E$. coli and with PhRelE monomer (Takagi et al. 2005, Neubauer et al. 2009). YoeB acts as a ribosome-dependent mRNA interferase (Christensen-Dalsgaard and Gerdes 2008). All experimental data suggest that YoeB inhibits protein synthesis, but its primary function as a blockade for this process is unclear. Whereas YoeB might act as a sequence-specific endoribonuclease or an mRNA interferase (Kamada and Hanaoka 2005, Christensen-Dalsgaard and Gerdes 2008), it could be that the endoribonuclease activity of YoeB is not primarily required for its inhibitory function for protein synthesis but to prevent the formation of the initiation complex (Zhang and Inouye 2009). In this latter case, YoeB would bind to the 50S ribosomal subunit in 70S ribosomes, and would interact with the A site, impairing formation of the initiation complex and, as a consequence, inhibiting protein synthesis. In turn, this inhibition would activate the latent endoribonuclease activity of either ribosomes or YoeB, resulting in cleavage of mRNA at the A site. Interaction of YoeB with its cognate antitoxin YefM could induce a conformational rearrangement of the RNase catalytic site of YoeB leading to the movement of some of the residues involved in catalysis, away from the active site. This conformational change in the catalytic site of YoeB could explain the mechanism employed by YefM to neutralize YoeB toxicity. Regulation of the expression of this TAS is similar to the other examples mentioned: YefM is the primary repressor and YoeB acts as a repressor enhancer (Kedzierska et al. 2007). However, this picture might be an oversimplification of the regulatory circuit (W. T. Chang and C. C. Yeo, personal communication).

\section{SOME POSSIBLE BIOMEDICAL APPLICATIONS/IMPLICATIONS OF TAS}

The structural and functional information available on TA interactions in several systems make it possible to search for or to design molecules able to interfere with these interactions and trigger the activity of the toxin. BRET (Bioluminiscence Resonance Energy Transfer) technology has been used to monitor toxin-antitoxin interactions (Nieto et al. 2006, Lioy et al. 2010). These assays could act as a powerful tool in the search for possible new antibiotics against cells containing endogenous or acquired toxin-antitoxin systems. The role of some TAS, particularly, the HipBA system of $E$. coli in bacterial persistence has been pointed out (Moyed and Bertrand 1983). A 10,000 increase in the numbers of persistent cells was associated with a mutation, hipA7, in the toxin gene. However, in spite of the well characterized mechanism of action of the toxin and of its neutralization by the antitoxin (Schumacher et al. 2009), the pathway involved in the activation of this epigenetic phenomenon, affecting a small fraction of the population, is not well understood. Persistent cells have important implications in combating antibiotic resistances in the clinical context as these cells are tolerant to antibiotics (Bigger 1944).

TAS were first characterized as virulenceassociated determinants on the basis of their prevalence in the chromosomes of virulent versus avirulent strains of specific human pathogens (Hopper 
et al. 2000, Daines et al. 2007). Moreover, TAS are widely conserved in plasmids from common hospital pathogens, including vancomycin-resistant enterococci (VRE) and aminoglycoside-resistant E. coli (Moritz and Hergenrother 2007, Perichon et al. 2008). The sequenced strains of Mycobacterium tuberculosis have 60 TAS including 7 homologues of mazEF (Pandey and Gerdes 2005). The contribution of these systems to the pathogenic character of this micro-organism remains to be fully explored. It is noteworthy that in bacterial species such as Vibrio cholerae, up to 13 TAS cluster in a mega-integron structure (Pandey and Gerdes 2005). Thus TAS could contribute to the genetic stability of mobile genetic elements and could play a role in stabilizing virulent traits during the evolution of pathogenic bacteria. Measurable attenuation of the virulence of pathogenic bacteria through the genetic inactivation of TAS using suitable animal infection models remains to be provided to properly address the possible role of TAS during infection.

Even before RNA was identified as the direct target of Kid, the bacterial Kid toxin was known to prevent proliferation in eukaryotic cells including yeast, oocites of Xenopus laevis and HeLa cells (de la Cueva-Méndez et al. 2003). Kid induced apoptosis in the later cells and the antitoxin Kis was protecting from these effects. This indicates that regulated expression of Kid and Kis might be used to kill particular cells, including tumour cell lines, in a selective way. This may be achieved by expressing the kid and kis genes under the control of promoters that are, respectively, induced and repressed in these cells, and that have the inverse behaviour in normal cells. Since Kid also inhibits the growth of embryonic cells, a similar strategy might be used to prevent the growth of particular cell lineages during development. This approach could have value in studies of differentiation, organogenesis or degenerative disorders (de la Cueva-Méndez et al. 2003). Indeed differential expression of the toxin and antitoxin genes of the parD system in fertilized embryos of zebrafish has been used to eliminate selectively the germ line of this fish and to study its role in sex differentiation (Slanchev et al. 2005). Similarly the RelE protein from $E$. coli acts as a global inhibitor of translation not only in bacteria or archaea but also in eukaryotic cells. In Saccharomyces cerevisiae the expression of the bacterial relE gene is toxic to yeast cells (Kristoffersen et al. 2000), and in a human osteosarcoma cell line the toxin RelE retards growth and leads to cell death by apoptosis (Yamamoto et al. 2002).

The growing structural and functional information on toxin-antitoxin systems open important avenues to the exploration of their biomedical and biotechnological implications and applications both in prokaryotic and eukaryotic cells.

\section{ACKNOWLEDGEMENTS}

The authors acknowledge the financial support of the MICINN, the Spanish Ministry of Science and Innovation, (CSD2008-00013; BFU200800179-E/BMC) and the dedication of many colleagues and friends that contributed to these studies over the years.

\section{REFERENCES}

Bernard P, Couturier M: Cell killing by the F plasmid CcdB protein involves poisoning of DNA-topoisomerase II complexes. J Mol Biol 226:735-745, 1992.

Bigger J: Treatment of Staphylococcal infections with penicillin by intermittent sterilisation. Lancet 244:497-500, 1944.

Bravo A, de Torróntegui G, Díaz R: Identification of components of a new stability system of plasmid $\mathrm{R} 1$, ParD, that is close to the origin of replication of this plasmid. Mol Gen Genet 210:101-110, 1987.

Bravo A, Ortega S, de Torróntegui G, Díaz R: Killing of Escherichia coli cells modulated by components of the stability system ParD of plasmid R1. Mol Gen Genet 215:146-151, 1988.

Buts L, Lah J, Dao-Thi MH, Wyns L, Loris R: Toxin-antitoxin modules as bacterial metabolic stress managers. Trends Biochem Sci 30:672-679, 2005.

Cherny I, Overgaard M, Borch J, Bram Y, Gerdes K, Gazit E: Structural and thermodynamic characterization of the Escherichia coli RelBE toxin-antitoxin system: indication for a functional role of differential stability. Biochemistry 46:12152-12163, 2007.

Christensen SK, Gerdes K: RelE toxins from Bacteria and Archaea cleave mRNAs on translating ribosomes, which are rescued by tmRNA. Mol Microbiol 48:1389-1400, 2003.

Christensen SK, Mikkelsen M, Pedersen K, Gerdes $\mathrm{K}$ : RelE, a global inhibitor of translation, is activated during nutritional stress. Proc Natl Acad Sci USA 98:14328-14333, 2001.

Christensen-Dalsgaard M, Gerdes K: Translation affects YoeB and MazF messenger RNA interferase activities by different mechanisms. Nucleic Acids Res 36:6472-6481, 2008. 
Condon C: Shutdown decay of mRNA. Mol Microbiol 61:573-583, 2006.

Daines, DA, Wu MH, Yuan SY: VapC-1 of nontypeable Haemophilus influenzae is a ribonuclease. J Bacteriol 189:5041-5048, 2007.

de la Cueva-Méndez G, Mills AD, Clay-Farrace L, Díaz-Orejas R, Laskey RA: Regulatable killing of eukaryotic cells by the prokaryotic proteins Kid and Kis. EMBO J 22:246-251, 2003.

Diago-Navarro E, Kamphuis MB, Boelens R, Barendregt A, Heck AJ, van den Heuvel RH, Díaz-Orejas R: A mutagenic analysis of the RNase mechanism of the bacterial Kid toxin by mass spectrometry. FEBS J 276:4973-4986 2009a.

Diago-Navarro E, Mora L, Buckingham RH, Díaz-Orejas R, Lemonnier M: Novel Escherichia coli RF1 mutants with decreased translation termination activity and increased sensitivity to the cytotoxic effect of the bacterial toxins Kid and RelE. Mol Microbiol 71:66-78, 2009b.

Francuski D, Saenger W: Crystal structure of the antitoxin-toxin protein complex RelB-RelE from Methanococcus jannaschii. J Mol Biol 393:898-908, 2009.

Garza-Sánchez F, Gin JG, Hayes CS: Amino acid starvation and colicin D treatment induce A-site mRNA cleavage in Escherichia coli. J Mol Biol 378:505-519, 2008.

Gerdes K, Christensen SK, Lobner-Olesen A: Prokaryotic toxin-antitoxin stress response loci. Nature Rev Microbiol 3:371-382, 2005.

Gerdes K, Rasmussen PB, Molin S: Unique type of plasmid maintenance function: postsegregational killing of plasmid-free cells. Proc Natl Acad Sci USA 83:3116-3120, 1986.

Gotfredsen M, Gerdes K: The Escherichia coli relBE genes belong to a new toxin-antitoxin gene family. Mol Microbiol 29:1065-1076, 1998.

Gotfredsen M, Gerdes K: Toxin-antitoxin modules may regulate synthesis of macromolecules during nutritional stress. J Bacteriol 182:561-572, 2002.

Grady R, Hayes F: Axe-Txe, a broad-spectrum proteic toxin-antitoxin system specified by a multidrug-resistant, clinical isolate of Enterococcus faecium. Mol Microbiol 47:1419-1432, 2003.

Gronlund H, Gerdes K: Toxin-antitoxin systems homologous with relBE of Escherichia coli plasmid P307 are ubiquitous in prokaryotes. J Mol Biol 285:1401-1415, 1999.

Hargreaves D, Santos-Sierra S, Giraldo R. Sabariegos-Jareño R, de la Cueva-Méndez G, Boelens R, Díaz-Orejas R, Rafferty JB: Structural and functional analysis of the kid toxin protein from E. coli plasmid R1. Structure 10:1425-1433, 2002.

Hayes F: Toxins-antitoxins: plasmid maintenance, programmed cell death, and cell cycle arrest. Science 301:1496-1499, 2003.

Hiraga S, Jaffe A, Ogura T, Mori H, Takahashi H: F plasmid ccd mechanism in Escherichia coli. J Bacteriol 166:100-104, 1986.

Hopper S, Wilbur JS, Vasquez BL, Larson J, Clary S, Mehr IJ, Seifert HS, So M: Isolation of Neisseria gonorrhoeae mutants that show enhanced trafficking across polarized T84 epithelial monolayers. Infection Immun 68:896-905, 2000.

Kamada K, Hanaoka F: Conformational change in the catalytic site of the ribonuclease YoeB toxin by YefM antitoxin. Mol Cell 19:497-509, 2005.

Kamada K, Hanaoka F, Burley SK: Crystal structure of the MazE/MazF complex: molecular bases of antidote-toxin recognition. Mol Cell 11:875-884, 2003.

Kamphuis MB, Bonvin AM, Monti MC, Lemonnier M, Muñoz-Gómez A, van den Heuvel RH, Díaz-Orejas R, Boelens R: Model for RNA binding and the catalytic site of the RNase Kid of the bacterial parD toxin-antitoxin system. J Mol Biol 357:115-126, 2006.

Kamphuis MB, Monti MC, van den Heuvel RH, López-Villarejo J, Díaz-Orejas R, Boelens R: Structure and function of bacterial kid-kis and related toxin-antitoxin systems. Protein Pept Lett 14:113-124, 2007a.

Kamphuis MB, Monti MC, van den Heuvel RH, Santos-Sierra S, Folkers GE, Lemonnier M, Díaz-Orejas R, Heck AJ, Boelens R: Interactions between the toxin kid of the bacterial parD system and the antitoxins Kis and MazE. Proteins 67:219-231, 2007b.

Kedzierska B, Lian LY, Hayes F: Toxin-antitoxin regulation: bimodal interaction of YefM-YoeB with paired DNA palindromes exerts transcriptional autorepression. Nucleic Acids Res 35:325-339, 2007.

Korch SB, Contreras H, Clark-Curtiss JE: Three Mycobacterium tuberculosis Rel toxin-antitoxin modules inhibit mycobacterial growth and are expressed in infected human macrophages. J Bacteriol 191:1618-1630, 2009.

Kristoffersen P, Jensen GB, Gerdes K, Piskur J: Bacterial toxin-antitoxin gene system as containment control in yeast cells. Appl Environ Microbiol 66:5524-5526, 2000.

Kumar P, Issac B, Dodson EJ, Turkenburg JP, Mande SC: Crystal structure of Mycobacterium tuberculosis YefM antitoxin reveals that it is not an intrinsically unstructured protein. J Mol Biol 383:482-493, 2008. 
Li GY, Zhang Y, Inouye M, Ikura M: Structural mechanism of transcriptional autorepression of the Escherichia coli RelB/RelE antitoxin/toxin module. J Mol Biol 380:107-119, 2008a.

Li GY, Zhang Y, Inouye M, Ikura M: Inhibitory mechanism of Escherichia coli RelE-RelB toxin-antitoxin module involves a helix displacement near an mRNA interferase active site. J Biol Chem 284:14628-14636, 2009.

Li X, Yagi M, Morita T, Aiba H: Cleavage of mRNAs and role of tmRNA system under amino acid starvation in Escherichia coli. Mol Microbiol 68:462-473, 2008b.

Lioy VS, Rey O, Balsa D, Pellicer T, Alonso JC: A toxin-antitoxin module as a target for antimicrobial development. Plasmid 63:31-39, 2010.

Makarova KS, Wolf YI, Koonin EV: Comprehensive comparative-genomic analysis of Type 2 toxin-antitoxin systems and related mobile stress response systems in prokaryotes. Biol Direct 4:19, 2009.

Masuda Y, Miyakawa K, Nishimura Y, Ohtsubo E: chpA and chpB, Escherichia coli chromosomal homologs of the pem locus responsible for stable maintenance of plasmid R100. J Bacteriol 175:6850-6856, 1993.

Monti MC, Hernández-Arriaga AM, Kamphuis MB, López-Villarejo J, Heck AJ, Boelens R, Díaz-Orejas R, van den Heuvel RH: Interactions of Kid-Kis toxin-antitoxin complexes with the parD operator-promoter region of plasmid R1 are piloted by the Kis antitoxin and tuned by the stoichiometry of Kid-Kis oligomers. Nucleic Acids Res 35:1737-1749, 2007.

Moritz EM, Hergenrother PJ: Toxin-antitoxin systems are ubiquitous and plasmid-encoded in vancomycin-resistant enterococci. Proc Natl Acad Sci USA 104:311-316, 2007.

Moyed HS, Bertrand KP: hipA, a newly recognized gene of Escherichia coli K-12 that affects frequency of persistence after inhibition of murein synthesis. J Bacteriol 155:768-775, 1983.

Muñoz-Gómez AJ, Santos-Sierra S, Berzal-Herranz A, Lemonnier M, Díaz-Orejas R: Insights into the specificity of RNA cleavage by the Escherichia coli MazF toxin. FEBS Lett 567:316-320, 2004.

Muñoz-Gómez AJ, Lemonnier M, Santos-Sierra S, Berzal-Herranz A, Díaz-Orejas R: Rnase/anti-RNase activities of the bacterial parD toxin-antitoxin system. J Bacteriol 187:3151-3157, 2005.

Neubauer C, Gao YG, Andersen KR, Dunham CM, Kelley AC, Hentschel J, Gerdes, Ramakrishnan $\mathrm{V}$, Brodersen DE: The structural basis for mRNA recognition and cleavage by the ribosome-dependent endonuclease RelE. Cell 139:1084-1095, 2009.

Nieto C, Pellicer T, Balsa D, Christensen SK, Gerdes $\mathrm{K}$, Espinosa $\mathrm{M}$ : The chromosomal relBE2 toxin-antitoxin locus of Streptococcus pneumoniae: characterization and use of a bioluminescence resonance energy transfer assay to detect toxin-antitoxin interaction. Mol Microbiol 59:1280-1296, 2006.

Nieto C, Cherny I, Khoo SK, de Lacoba MG, Chan WT, Yeo CC, Gazit E, Espinosa M: The yefM-yoeB toxin-antitoxin systems of Escherichia coli and Streptococcus pneumoniae: functional and structural correlation. J Bacteriol 189:1266-1278, 2007.

Overgaard M, Borch J, Jorgensen MG, Gerdes K: Messenger RNA interferase RelE controls relBE transcription by conditional cooperativity. Mol Microbiol 69:841-857, 2008.

Pandey D, Gerdes K: Toxin- antitoxin loci are highly abundant in free-living but lost from host-associated prokaryotes. Nucleic Acids Res 55:78-89, 2005.

Pedersen K, Christensen SK, Gerdes K: Rapid induction and reversal of a bacteriostatic condition by controlled expression of toxins and antitoxins. Mol Microbiol 45:501-510, 2002.

Pedersen K, Zavialov AV, Pavlov MY, Elf J, Gerdes $\mathrm{K}$, Ehrenberg M: The bacterial toxin RelE displays codon-specific cleavage of mRNAs in the ribosomal A site. Cell 112:131-140, 2003.

Perichon B, Bogaerts P, Lambert T, Frangeul L, Courvalin P, Galimand M: Sequence of conjugative plasmid pIP1206 mediating resistance to aminoglycosides by $16 \mathrm{~S}$ rRNA methylation and to hydrophilic fluoroquinolones by efflux. Antimicrob Agents Chemother 52:2581-2592, 2008.

Pimentel B, Madine MA, de la Cueva-Méndez G: Kid cleaves specific mRNAs at UUACU sites to rescue the copy number of plasmid R1. EMBO J 24:3459-3469, 2005.

Potrykus K, Santos S, Lemonnier M, Díaz-Orejas R, Wegrzyn G: Differential effects of Kid toxin on two modes of replication of lambdoid plasmids suggest that this toxin acts before, but not after, the assembly of the replication complex. Microbiology 148:2489-2495, 2002.

Ruiz-Echevarría MJ, de la Cueva G, Díaz-Orejas: Translational coupling and limited degradation of a polycistronic messenger modulate differential gene expression in the parD stability system of plasmid R1. Mol Gen Genet 248:599-609, 1995a. 
Ruiz-Echevarría MJ, de la Torre MA, Díaz-Orejas R: A mutation that decreases the efficiency of plasmid R1 replication leads to the activation of parD, a killer stability system of the plasmid. FEMS Microbiol Lett 130:129-135, 1995b.

Santos-Sierra S, Giraldo R, Díaz-Orejas R: Functional interactions between homologous conditional killer systems of plasmid and chromosomal origin. FEMS Microbiol Lett 152:51-56, 1997.

Santos-Sierra S, Giraldo R, Díaz-Orejas R: Functional interactions between chpB and parD, two homologous conditional killer systems found in the Escherichia coli chromosome and in plasmid R1. FEMS Microbiol Lett 168:51-58, 1998.

Schumacher MA, Piro KM, Xu W, Hansen S, Lewis K, Brennan RG: Molecular mechanisms of HipA-mediated multidrug tolerance and its neutralization by HipB. Science 323:396-401, 2009.

Slanchev K, Stebler J, de la Cueva-Méndez G, Raz E: Development without germ cells: the role of the germ line in zebrafish sex differentiation. Proc Natl Acad Sci USA 102:4074-4079, 2005.

Takagi H, Kakuta Y, Okada T, Yao M, Tanaka I, Kimura M: Crystal structure of archaeal toxin-antitoxin RelE-RelB complex with implications for toxin activity and antitoxin effects. Nat Struct Mol Biol 12:327-331, 2005.

Tsilibaris V, Maenhaut-Michel G, Mine N, Van Melderen L: What is the benefit to Escherichia coli of having multiple toxin-antitoxin systems in its genome? J Bacteriol 189:6101-6108, 2007.

Tsuchimoto S, Ohtsubo H, Ohtsubo E: Two genes, pemK and pemI, responsible for stable maintenance of resistance plasmid R100. J Bacteriol 170:1461-1466, 1988.

Tsuchimoto S, Nishimura Y, Ohtsubo E: The stable maintenance system pem of plasmid R100: degradation of PemI protein may allow PemK protein to inhibit cell growth. J Bacteriol 174:4205-4211, 1992.

Van Melderen L, Saavedra De Bast M: Bacterial toxin-antitoxin systems: more than selfish entities? PLoS Genet 5:e1000437, 2009.

Yamamoto TA, Gerdes K, Tunnacliffe A: Bacterial toxin RelE induces apoptosis in human cells. FEBS Lett 519:191-194, 2002.

Yoshizumi S, Zhang Y, Yamaguchi Y, Chen L, Kreiswirth BN, Inouye M: Staphylococcus aureus YoeB homologues inhibit translation initiation. J Bacteriol 191:5868-5872, 2009.

Zhang J, Zhang Y, Zhu L, Suzuki M, Inouye M: Interference of mRNA function by sequence-specific endoribonuclease PemK. J Biol Chem 279:20678-20684, 2004.

Zhang Y, Inouye M: The inhibitory mechanism of protein synthesis by YoeB, an Escherichia coli toxin. J Biol Chem 284:6627-6638, 2009.

Zhang Y, Zhang J, Hoeflich KP, Ikura M, Qing G, Inouye $\mathrm{M}$ : MazF cleaves cellular mRNAs specifically at ACA to block protein synthesis in Escherichia coli. Mol Cell 12:913-923, 2003. 